\title{
Incomplete Protocol Artifact
}

National Cancer Institute

\section{Source}

National Cancer Institute. Incomplete Protocol Artifact. NCI Thesaurus. Code C87046.

An image of reduced quality resulting from the use of instructions with missing steps. 